\title{
PathF3 - Improving accuracy and network load in available bandwidth estimation based on SLoPS Methodology
}

\author{
Mazni Amil, Mohamad Yusoff Alias, and Khairil Anuar
}

\begin{abstract}
In this paper we presented the results of a series of available bandwidth experiments conducted in a control environment testbed by using WAN Emulator to represent the actual network. The approaches are classified according to the available bandwidth methodology of Self Loading Periodic Streams (SLoPS). The comparative analysis of state-of-the-art available bandwidth will be focusing on a tool called Pathload that used SLoPS methodology, and compare it with our new technique called PathF3. The results show that PathF3 can give a near accurate estimation as compared to Pathload
\end{abstract}

Index Terms - PathF3, Available Bandwidth, Bandwidth Measurement and SLoPS

\section{INTRODUCTION}

With the increasing penetration of Internet access, there has been an increasing interest in bandwidth estimation development such as capacity and available bandwidth, bulk transfer capacity or achievable throughput.. In this paper, we proposed a new technique based on Self Loading Periodic Streams (SLoPS) call PathF3). The PathF3 naming is based on 3 fleets that are used in PathF3 while sending the packet from Sender $(\mathrm{S})$ to Receiver $(\mathrm{R})$ This technique will improve the accuracy and network load performance that Pathload technique already faced. Results of the experiment between PathF3 and Pathload shows that our technique give better output.

The available bandwidth is the maximum IP-layer (Internet Protocol Layer) throughput that a flow can get in the path from $\mathrm{S}$ to $\mathrm{R}$ ), without reducing the data rate for the rest of the traffic in the same path. Prasad et al. stated that the available bandwidth is depends not only on the link capacity, but also on the traffic load, and is typically a time-varying metric [1].

Available bandwidth $\mathrm{A}_{\mathrm{i}}$ of a hop $I$ of an end-to-end link over a certain time interval is given by the unutilized fraction of capacity:

Manuscript received July 20, 2009

Mazni Amil is with the Telekom Research and Development : TMR\&D Innovation Centre, Lingkaran Teknokrat Timur, 63000 Cyberjaya, Selangor, Malaysia (phone: (603) 88839331; fax: (603) 88839596; e-mail: mazni@tmrnd.com.my).

Mohamad Yusoff Alias, is with Engineering Faculty in Multimedia University, Jalan Multimedia 63100 Cyberjaya Selangor Malaysia (e-mail: yusoff@mmu.edu.my).

Khairil Anuar is with Engineering Faculty in Multimedia University, Jalan Multimedia 63100 Cyberjaya Selangor Malaysia (e-mail: khairil@mmu.edu.my).

$$
A_{i}=\left(1-u_{i}\right) C
$$

where $u_{i}$ is the average utilization of hop $I$ in the given time interval, and the $\mathrm{C}_{\mathrm{i}}$ is the capacity of hop $i$. By extending the available bandwidth definition to an $H$-hop path, the authors define the available bandwidth of the end-to-end path, A, as the minimum available bandwidth of all $H$ hops

$$
\begin{gathered}
A=\min A_{i} . \\
i=1, \ldots, H
\end{gathered}
$$

The hop with the minimum available bandwidth is called the tight link of the end-to-end path. Self-Loading Periodic Streams (SLoPS) are one of the famous methodologies for available bandwidth measurement. SLoPS implementation require access at both ends of path Sender (S) and Receiver (R). Sender sends series of packets to the Receiver at the stream rate $R$ of larger than the available bandwidth. Every packet gets a timestamp at sender side. Comparing the difference of successive packets timestamp and their arrival times can infer the available bandwidth. If the stream rate $R$ is higher than the available bandwidth, the one-way delays of successive packets at the receiver show an increasing trend as shown in Figure 1.
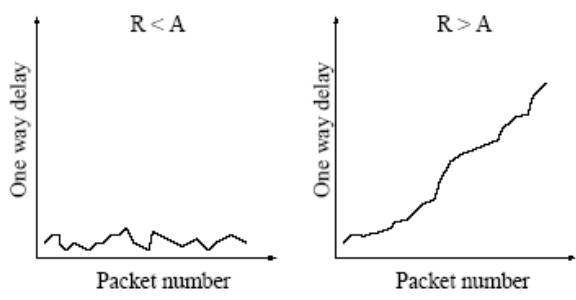

Figure 1. One way delays increase when the stream rate $R$ is larger than the available bandwidth $A$, but do not increase when $R$ is lower than $A$ [1]

\section{MOTIVATION}

The motivation to researcher and developer to use bandwidth are encouragement from software requirement, network traffic and methodology implementation.

There is a trend from software (what software?) especially related to Internet usage to get an information about bandwidth parameters such as capacity and available bandwidth, bulk transfer capacity or achievable throughput, as suggested by Lai and Baker [7], "Accurate network bandwidth measurement is important to a variety of network applications". These values somehow get an input from data entry (manually enter by end-user or administrator), internal 
system, or interface to external software, database or even hardware. For an example, before customer can download whatever file(s), the system tried to ask customer to choose and select an internet connection speed they have or if user want to watch a movie via streaming. User need select and choose appropriate bit-rate based on their internet connection speed. But, now a days there is an effort to develop [3]-[4] the software (i.e. video player and server) which can received or stream the scalable media content with different bit-rate according to their respected bandwidth. So, in fulfilling the software requirement, an estimation of the bandwidth with easy, fast, accurate and low traffic will be attached to the application software.

Internet Service Provider (ISP) such as Telekom Malaysia Berhad has implemented wireless, high speed and next generation networks. These networks are being constructed in-order to support the large growth of the Internet population, as well as enabling higher bandwidth services to run over the network. There is an increasing demand in knowing either the performance obtained from these networks is up to the lever expected from them [5]. So, the ISP needs a tool to measure the bandwidth and troubleshoot the problem if the required bandwidth value is below the expected level. Beside the ISP, customers also need a tool to monitor their network subscription. Thus, it is create a demand in developing tools that enable customers to access the performance without any assistance. These monitoring tools need perform quickly, easily and with high accuracy in measuring the end-to-end performance of the probe network.

For ISP, the tool that measures the performance such as data rate or congestion control is needed. It should be noted that different network technologies may implement rate control at different levels [6]. Referring to [8], the traffic in a network is the data flowing in an encoded form between pairs of points within it. These points may be simply the end points of a single transmissions link, a client/server pair in a local area network or two hosts joined by a wide area network or internet. So there is a need of a close inspection of figures resulting from a period of traffic measurement in a network reveals an interesting inconsistency. A key feature in providing the right service is an awareness of what customers want in terms of the information they wish to send and receive. The slightest misunderstanding such as the nature of the traffic the network carries can be costly.

The third motivation is the accuracy of bandwidth estimation. The estimation need to be fast, easy, won't congest the network and more the most important thing it must be very accurate. There are several methodologies, studies and industrial deployment has introduced their implementations. All methodologies exhibit the disadvantages and advantages between them. Although, all bandwidth estimation methodologies gives an exact metric but it may not be accurate for different networks or situations. For an example, the technique to calculate the available bandwidth point-to-point for wireless 3G (Third Generation of Mobile Network) may not be the same as, to compare with, in local or wide area network using fiber optic or Bluetooth.

\section{RELATED WORKS}

Pathload is a tool for estimating the available bandwidth of an end-to-end path from a host sender to a host receiver. Pathload was designed as an open source and the source code could be downloaded at [9]. Briefly, Pathload is based on the following key idea: When a process at Sender sends a periodic stream of UDP (User Datagram Protocol) packet at a rate higher than the available bandwidth in the path, the relative one-way packet delays will show an increasing trend of stream rate. When the stream rate is lower than the available bandwidth, the relative one-way packet delays show no consistent trend. From that point Pathload estimate the available bandwidth when the one-way packet delays start show the un-consistent trend.

Vinay J. Ribeiro et al (2003) introduced PathChirp as an active probing tool for estimating the available bandwidth on a communication network path. It's an open source, UNIX dependent and available for download at [10]. Based on the concept of "self-induced congestion," PathChirp features an exponential flight pattern of probes that can be called as a chirp. Packet chips offer several significant advantages over current probing schemes based on packet pairs or packet trains. By rapidly increasing the probing rate within each chirp, PathChirp obtains a rich set of information from which it can dynamically estimate the available bandwidth.

STAB(Spatio-Temporal Available Bandwidth) is an extension of PathChirp, introduced by [12]. STAB is an active probing tool for locating thin links on a network path. A thin link is a link with less available bandwidth than all links preceding it on the path. The last thin link on the path is the link with the minimum available bandwidth or tight link. $S T A B$ combines the concept of "self-induced congestion", the probing technique of "packet tailgating", and special probing trains called "chirps" to efficiently locate the thin links. The advantage of STAB as compared to PathChirp is that STAB is not only able to estimate the available bandwidth but is also to locate the thin links on a network path.

For this research, the focus was on Pathload and comparing it with a new technique develops in house.

\section{PATHF3 KeY IDEA}

The new technique is based on the idea of SLoPS methodology [1,4]. PathF3 name was chosen based on the three fleets sending from sender to receiver during the packet stream. SLoPS methodology is one-way delay of a periodic packet stream that showed an increasing trend when the stream's rate is higher than the available bandwidth.

PathF3 is an enhanced version of Pathload in order to improve the network performance and accuracy of the existing Pathload. The improvement was done by looking at the total number of fleets and packet size that are streamed per train. Existing Pathload sends multiple train of 1200 same sized packet with a total size of 240,000 bytes per stream. 
On the other hand, PathF3 was designed by sending the multiple trains with 180 same sized packets with total bytes of 36,000 bytes per stream. The calculation for the total number of bytes is given by:

Total Bytes $=$ Number of Fleets $x$ Total Packets $x$ Bytes per Packet

During experiment's measurement, PathF3 generates report in range format rather than single estimation. The range estimates the variation of available bandwidth while the average of available bandwidth shows from the centre of the range.

PathF3 consists of two programs namely sender and receiver. The sender is installed on source host, while the receiver is installed on destination host. PathF3 technique requires access to both ends of the path, but does not require super user privileges because it only sends UDP packets.

Before the sender sends the packet to the receiver, the sender will open a connection to receiver. The connection will be established between the sender and the receiver in order to make sure the sender could send the packet and the receiver could calculate the packet stream.

When the sender sends a periodic stream of UDP packet at a rate higher than the available bandwidth in the path, the relative one-way packet delays show an increasing trend. When the stream rate is lower than the available bandwidth, the relative one-way packet delays show no consistent trend. During an estimation process, the sender transmits trains of probe packets at different rates, while the receiver collects the one-way transmission delays (OWDs) of the packet trains and processes the OWDs with statistic algorithm to infer available bandwidth.

From that point, PathF3 will estimate the available bandwidth when the one-way packet delays start to show an inconsistent trend.

\section{PathF3 BASEd on SLoPS Methodology}

In this section, the SLoPS measurement methodology is described. PathF3 is designed based on SLoPS methodology. A periodic stream in SLoPS consists of $K$ packet of size $L$, sent to the path at a constant rate $R$. If the stream rate $R$ is higher than the available bandwidth, then the one-way delays of successive packets at the receiver show an increasing trend. In this section the research illustrate this fundamental effect in its simplest form through an analytical model with stationary and fluid cross traffic.

\section{A. SLoPS with Fluid Cross Traffic}

Consider a path from Sender $(\mathrm{S})$ to Receiver $(R)$ that consists of $H$ links, $i=1 \ldots H$. The capacity of link $i$ is $C_{i}$. In this study, a stationary (i.e., time invariant) and fluid model for the cross traffic in the path are being used. So, if the available bandwidth at link $i$ is $A_{i}$, the utilization is $u_{i}=\left(C_{i}-A_{i}\right) / C_{i}$ and there are $u_{i} C_{i}{ }^{T}$ bytes of cross traffic departing from and arriving at link $I$ in any interval of length $T$.This will also, assume that the links follow the first-come-first-served queuing discipline, ${ }^{1}$ and that they are adequately buffered to avoid losses. Here, any propagation or fixed delays in the path were ignored, as they do not affect the delay variation between packets. The available bandwidth $A$ in the path is determined by the tight $\operatorname{link}^{2} t \in\{1, \ldots, H\}$ with

$$
A_{t}=\min _{i=1 \cdots H} A_{i}=\min _{i=1 \cdots H} C_{i}\left(1-u_{i}\right)=A .
$$

Suppose that $S$ sends a periodic stream of $K$ packets to $R$ at a rate $R_{0}$, starting at an arbitrary time instant. The packet size is $L$ bytes, and so packets are sent with a period of $T=L / R_{0}$ time units. The one-way delay (OWD) $D^{k}$ from $S$ to $R$ of packet $k$ is

$$
D^{k}=\sum_{i=1}^{H}\left(\frac{L}{C_{i}}+\frac{q_{i}^{k}}{C_{i}}\right)=\sum_{i=1}^{H}\left(\frac{L}{C_{i}}+d_{i}^{k}\right)
$$

Where $q_{i}^{k} \quad$ is the queue size at link $i$ upon the arrival of packet $k\left(q_{i}^{k}\right.$ does not include packet $\left.k\right)$, and $d_{i}^{k}=q_{i}^{k} / C_{i}$ is the queuing delay of packet $k$ at link $i$. The OWD difference between two successive packets $k$ and $k+1$ is

$$
\begin{gathered}
\Delta D^{k} \equiv D^{k+1}-D^{k}=\sum_{i=1}^{H} \frac{\Delta q_{i}^{k}}{C_{i}}=\sum_{i=1}^{H} \Delta d_{i}^{k} \\
\text { where } \Delta q_{i}^{k}=q_{i}^{k+1}-q_{i}^{k} \text { and } \Delta d_{i}^{k}-\Delta q_{i}^{k} / C_{i}
\end{gathered}
$$

It can now show that if $R_{0}>A$, then the $K$ packets of the periodic stream will arrive at $R C V$ with increasing OWDs, while if $R_{0} \leq A$ the stream packets will encounter equal OWDs.

This property is stated: Proposition 1: If $R_{0}>A$, then $\Delta D^{k}>$ 0 for $k=1, \ldots K-1$. Else, if $R_{0} \leq A, \Delta D^{k}=0$ for $k=1, \ldots K-1$.

One may think that the available bandwidth $A$ can be computed directly from the rate at which the stream arrives at $R$. This is the approach followed in packet train dispersion techniques.

Proposition 2: The rate of $R_{H}$ the packet stream at $R$ is a function, in the general case, of $C_{i}$ and $A_{i}$ for all $i=1, \ldots, H$.

${ }^{1}$ In links with per-flow or per-class queuing, SLoPS can monitor the sequence of queues that the probing packets go through.

${ }^{2}$ If there are more than one links with available bandwidth A, the tight link is the first of them, without loss of generality.

\section{B. Iterative Algorithm to Measure}

Based on Proposition 1, an iterative algorithm for the end-to-end measurement of $A$ can be constructed. Suppose that $S$ sends a periodic stream $n$ with rate $R(n)$. The receiver analyzes the OWD variations of the stream, based on Proposition 1, to determine whether $R(n)>A$ or not. Then, $R$ notifies $S$ about the relation between $R(n)$ and $A$. If , $R(n)>A$ $S$ sends the next periodic stream $(n+1)$ with rate $R(n+1)<$ $R(n)$. Otherwise, the rate of $\operatorname{stream}(n+1)$ is $R(n+1)>R(n)$. Specifically $R(n+1)$, can be computed as follows: 


$$
\begin{array}{rlrl}
\text { If } R(n) & >A, & & R^{\max }=R(n) \\
\text { If } R(n) & \leq A, & R^{\min }=R(n) \\
R(n+1) & =\left(R^{\max }+R^{\min }\right) / 2 .
\end{array}
$$

$R^{\min }$ and $R^{\max }$ are lower and upper bounds for the available bandwidth after stream, respectively. Initially $R^{\min =0}$, and $R^{\max }$ $=$ can be set to a sufficiently high value $R_{0}^{\max }>A^{3}$ The algorithm terminates when $R^{\max }-R^{\min } \leq w$, where $w$ is the user-specified estimation resolution. If the available bandwidth $A$ does not vary with time, the previous algorithm will converge to a range $\left[R^{\min }, R^{\max }\right]$ that includes $A$ after $\left[\log _{2}\right.$ $\left.R_{0}^{\max } / w\right]$ streams.

\section{SLoPS With Real Cross Traffic}

So far assumption had been made that the available bandwidth $A$ is constant during the measurement process. In reality, the available bandwidth may vary because of two reasons. First, the available bandwidth $A^{\Gamma}(t)$ process may be non-stationary, and so its expected value may also be a function of time. Even if $A^{\Gamma}(t)$ is stationary, however, the process $A^{\Gamma}$ can have a significant statistical variability around its (constant) mean $E\left[A^{\Gamma}\right]$, and to make things worse, this variability may extend over a wide range of timescales $\Gamma$.

\section{EXPERIMENTAL DESIGN}

For the experimental setup, it had been decided to run the experiment at Multimedia Lab in TM Research and Development. The experiment also implement a few situation, scenario and run with the different time to get a better results. The comparative analysis of the available bandwidth was focusing on tools that using SLoPS methodology such as Pathload. Below are the experimental setups and scenario for the Available Bandwidth Estimation testing.

\section{A. Experiment 1: End-to-End without traffic}

The first set-up is for the end-to-end network without any traffic. In this environment, two desktops running Linux Ubuntu 7.0.3 operating systems are connected through the wide area network (WAN) Emulator as shown in Figure 2. By setting the bandwidth at WAN Emulator as scenario below and packet from sender to receiver was sent to get the result for available bandwidth at that time. Environment test is a control environment by simulating the network using the WAN Emulator and by assuming that there is no traffic between the two hosts. To get better results, it were tested for each tools for 50 times with the same bandwidth setting. The bandwidths that have been set at WAN Emulator for the testing purpose were $512 \mathrm{kbps}$, $1 \mathrm{Mbps}$, 2Mbps and 4Mbps

\section{B. Experiment 2: End-to-End with Wireshark}

The second experiment captured the result for the range of bandwidth estimation while opening the Wireshark. Wireshark is a tool for Monitoring Network Performance. For this environment, also it uses two desktops running Linux Ubuntu 7.0.3 operating system connected through the WAN Emulator as shown in Figure 2. The bandwidth was set at
WAN Emulator. Environment test is considered local and it is within a controlled environment. To get better results, test had been done for each tools for 50 times with the same bandwidth setting. Just as in Experiment 1, the bandwidths that had been set at WAN Emulator for the testing purpose are 512kbps, $1 \mathrm{Mbps}, 2 \mathrm{Mbps}$ and $4 \mathrm{Mbps}$

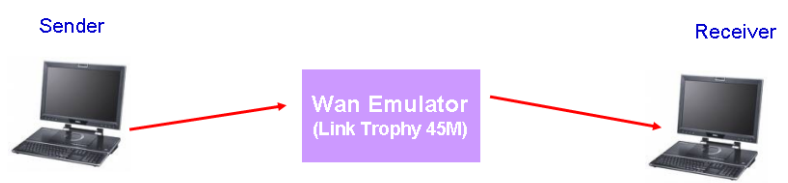

Figure 2 . Experiment setup for the wired environment without traffic and with Wireshark

\section{EXPERIMENTAL RESULTS}

\section{A. Result for Experiment 1}

Figure 3 shows the comparison bar chart for average results in Experiment 1. From Figure 3, it can be seen that PathF3 is more consistence and more accurate in estimating the available bandwidth as compared to Pathload. For the $4 \mathrm{Mbps}$ bandwidth, PathF3 result shows a range of 3.68-3.73 Mbps available bandwidth measured from this technique. If look closely at the 2 Mbps bandwidth, PathF3 proves that the available bandwidth measurement is nearer to the bandwidth offered. When compare the result for Pathload, the result range is $0.00-1.98 \mathrm{Mbps}$ and it means the available bandwidth measured for $2 \mathrm{Mbps}$ is 0 , which could not be accepted because the experiment was in a control network without any traffic interruption.

For the $512 \mathrm{kbps}$ bandwidth, it is proven that PathF3 could handle for measurement less than $1 \mathrm{Mbps}$ as compared to Pathload that cater only the bandwidth that is greater than 1 Mbps. From the results captured, it could be summarized that PathF3 has successfully given a close approximate measurement of the available bandwidth as per propose in this research.

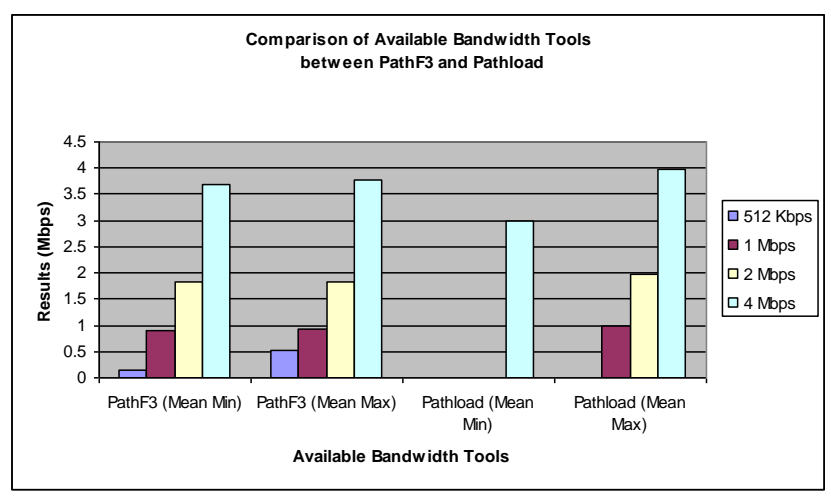

Figure 3 . The average result from Experiment 1

\section{B. Result for Experiment 2}

Figure 4 show the comparison between PathF3 and Pathload in terms of Network performance experience and the results show in average bytes/second.

From result show for $2 \mathrm{Mbps}$ the average bytes/second are 9686.25 send from sender to receiver using PathF3, compare 
to Pathload 11026.58. This results shows that PathF3 sends less bytes/second than Pathload. From the results shows at Figure 3 proved that PathF3 techniques help to reduce the network load in the network environment.

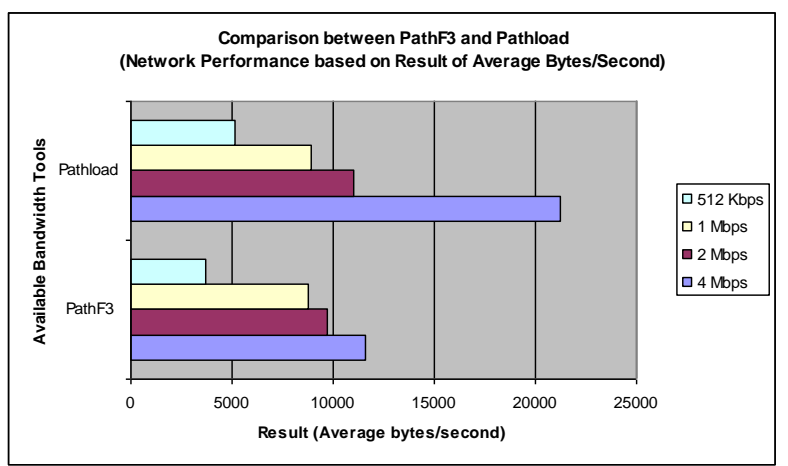

Figure 4. The average result from Experiment 1

\section{CONCLUSION AND FutURE WORK}

This paper had presented the experimental results and findings that PathF3 has successfully measured end-to-end available bandwidth in Linux Platform as compared to Pathload. This experiment had shown the decreasing of the network load and also improvement accuracy of the result. At bandwidth set to $512 \mathrm{kbps}$, it is found that Pathlaod is ineffective and cannot be trusted. Whereas the PathF3 measurement is more reliable and consistent. For future plan, experiment will be done in Windows operating system platform. Also further improvement will be done for PathF3 to give the better accuracy, more reliability and consistent results as compared to Pathload..

\section{REFERENCES}

[1] Prasad. R., Dovrolis, C., Murray, M., and Claffy, K., "Bandwidth Estimation: Metrics, Measurement Techniques, and Tools", IEEE Network, vol. 17, Issue 6, pp. 27-35, Nov.-Dec. 2003.

[2] C. Dovrolis and M. Jain, "End-to-End Available Bandwidth: Measurement Methodology, Dynamics, and Relation with TCP Throughput," IEEE/ACM Transactions in Networking, August 2003.

[3] Feng Wu, Shipeng Li, Rong Yan, Xiaoyan Sun and Ya-Qin Zhang, "Efficient And Universal Scalable Video Coding", Volume 11, p.p. 37 40, IEEE ICIP 2002.

[4] Guillaume Boisson , Edouard François and Christine Guillemot, "Efficient Scalable Motion Coding For Wide-Range Scalable Video Compression", Proceedings of XII European Signal Processing Conference, p.p. 1979-1982, Vienna, September 2004.

[5] James Curtis and Tony McGregor, "Review of Bandwidth Estimation Techniques", Department of Computer Science, University of Waikato, Hamilton, New Zealand.

[6] John G. Apostolopoulos, Wai-Tian Tan and Susie J. Wee, Video "Streaming: Concepts, Algorithms, And Systems, Technical Report", Mobile And Media Systems Laboratory, Hewlett-Packard 2002.

[7] Lai. K. and Baker M., "Measuring bandwidth", Proceedings of 18th Annual Joint Conference of the IEEE Computer and Communications Societies. Volume 1, p.p. 235 - 245, 21-25 Mar 1999.

[8] Vic Grout, "Measuring Network Traffic: The Hidden Distribution", Mathematics Today: Bulletin of the Institute of Mathematics and its Applications, Volume 37, Number 6, p.p.179-183, December 2001.

[9] C. Dovrolis and M. Jain "A measurement tool for end-to-end available bandwidth" This work was supported by the SciDAC program of the US Department of Energy (award \# DE-FC02- 01ER25467).

[10] Vinay J. Ribeiro, Rudolf H. Riedi, Richard G. Baraniuk Jiri Navratil,, Les Cottrell "PathChirp: Efficient Available Bandwidth Estimation for Network Paths", 2003.

[11] PathChirp tool, available http://www.spin.rice.edu/Software/pathChirp/

\section{[12] Stab tool, available at http://www.spin.rice.edu/Software/STAB/}

Mazni Amil obtained the Bachelor in Information Science Technology from National University of Malaysia in August 1998. Currently she is the second year student in Multimedia University Malaysia in Master of Engineering Science. She is currently an Associate Senior Researcher in the Telekom Research and Development. Her research interests cover the field of Networking especially on Network Management and Network performance.

Mohamad Yusoff Alias obtained the B.S. degree in engineering (electrical engineering) from the University of Michigan, Ann Arbor, in May 1998. He then received his Ph.D. degree in December 2004 from the School of ECS, University of Southampton in the UK. He is currently a Senior Lecturer in the Faculty of Engineering, Multimedia University in Malaysia. His research interests cover the field of wireless communications, especially in OFDM, multiple-antenna systems, multiuser detection, genetic algorithms in communications, and multimedia applications

Khairil Anuar graduated from the University of Salford, United Kingdom with BEng (Hons) in Electrical and Electronics Engineering in 1998. He then pursued his Master of Science (Telecommunication Engineering) degree at the National University of Malaysia, Malaysia and graduated in 2004 . He is currently attached to Multimedia University, Malaysia as a lecturer and researcher. His research interests cover the area of wireless networking and wireless sensor network. 